\title{
Paradox of Multi Criteria Decision Making Processes
}

\author{
Gil Greenstein \\ Faculty of Technology Management, Holon Institute of Technology (H.I.T), Israel
}

Copyright $(2017$ by authors, all rights reserved. Authors agree that this article remains permanently open access under the terms of the Creative Commons Attribution License 4.0 International License

\begin{abstract}
In the global environment decision makers should considers several aspects. Some of them have contradicted influence, and some aspects are not comparable to others. It leads decision-makers to limited ability of judgment between several aspects. Moreover, in a vibrant world, where technological changes occur in small intervals over time, the considerations of decision makers are changing frequently and dramatically. Thus, decision makers cannot adjust their decision mechanism instantly. This leads us to the need to better understand human decision process. This research presents a new extension of the Information Structure model, which is built using a normative approach in order to understand the mechanisms of multi-criteria decision-making (MCDM) processes. A consequent analysis explains decision-making behavior under the assumptions of bounded rationality. The analysis deals with decision situations, in which it is assumed that a decision-maker cannot formulate a joint utility function constituted from different criteria, which are completely or partially inestimable (intangible). Moreover, the model demonstrates problematic issues of MCDM processes; for example, when the improvement of a service paradoxically leads to a decline in a firm's market share. The model enables implementation of the general informativeness ratio between information structures to such environments. The model is based on the assumption of Bounded-Rationality, that a decision process takes several criteria into account, and handles with a decision-maker that cannot formulate a joint utility function constituted from different criteria. The results of the analysis after applying the model suggest that an optimal solution cannot be obtained; thus, a different approach is proposed that of optimization subject to rational satisfaction. This approach can be implemented, for example in the design stage of decision support systems (DSS). The method may be implemented for decision situations, in which intangible decision criteria are taken into consideration.
\end{abstract}

Keywords Multi Criteria Decision Making, Decision Support Systems, Bounded Rationality, Information Technology Management

\section{Introduction}

\subsection{Background and Motivation}

In the global environment, decision-makers should be aware of two facts: some aspects have a contradictory influence, and some aspects are not comparable to others. This provides decision-makers with only a limited sense of good judgment when it comes to differentiating among several aspects. Moreover, in a dynamic world, where rapid technological changes are constantly occurring, decision-makers' considerations change frequently and dramatically. Thus, decision-makers cannot always be instantly adjusting and updating their decision mechanism. This demands a better understanding of the human decision process. Savage's [24] rationality assumptions are the basis for calculating the value of information by using the information structure model [15]. Moreover, rationality assumptions [24] propose that a decision-maker can make his decisions instantly and calculate utility using a single utility function, which considers each and every aspect. But is this actually possible?

Traditional Utility Theory suggests an optimization method. This method, however, is not always appropriate in the modern decision-making environment. This research presents a new type of decision-maker: an optimizer subject to rational satisfaction. Such a decision-maker does not attempt to evaluate an optimal expected utility. She, rather, makes her decision based on a minimal satisfaction level. This research proves that there is equilibrium in the case of a two-criterion utility function. The presented analytical model is an extension of the Information Structure Model (another way of presenting decision trees), which demonstrates the dual paradox of decision-makers in a multi-criteria decision situation.

\subsection{Research Objectives}

This research presents an analytical model, which is built using a normative approach in order to understand the mechanisms of multi-criteria decision-making processes. A consequent analysis explains the decision maker's behavior under the assumptions of bounded rationality. The analysis 
deals with decision situations, in which it is assumed that a decision-maker cannot formulate a joint utility function constituted from different criteria, which are completely or partially inestimable. Moreover, the model reveals the problematic issues of multi-criteria decision-making processes; for example, when the improvement of a service paradoxically leads to a decline in a firm's market share.

The presented model is an extension of the Information Structure Model to a multi-criteria (multi-objective) decision environment. Moreover, the model enables implementation of the general informativeness ratio [15] between information structures to a multi-criteria decision situation. Multi-criteria decision processes, where decision-makers cannot use a "standard" utility function, are modeled. Furthermore, the model presents a new type of decision-maker: an optimizer, which is subject to rational satisfaction, and in which elements of bounded rationality are integrated into the model. The elements of a decision-maker's bounded rationality are based on the following assumptions: She deals with intangible criteria that cannot be measured quantitatively

Hence, such a decision-maker does not attempt to evaluate an overall expected utility.

She makes her decision based on a max-min satisfaction level for each criterion separately.

\subsection{Research Structure}

The next section contains a literature review in the area of multi-criteria decision-making and the value of information. In Section 3, a new type of multi-objective criteria decision-maker - "an optimizer subject to rational satisfaction" - is presented. Section 4 demonstrates the paradoxical implications generated by this type of decision-making. Section 5 contains a summary and conclusions; the proof of a theorem appears in Appendix 1.

\section{A review of Multi Criteria Decision Making and the Value of Information}

The third rationality assumption is based on a decision-maker's infinite calculation ability - whether she can combine different criteria in a joint utility function. Furthermore, traditional Decision Theory assumes that a decision-maker can identify an optimal decision, although some aspects of bounded rationality may cause her to make a decision with satisfaction-related expected outcomes [23] [25]. Take, for example, the following conflict in a decision situation: There are several criteria related to the decision-making process and the decision-maker cannot rank them. Hence, Bounded Rationality leads to a satisfactory decision, rather than an optimal one.

In fact, in contrary to optimizing (achieving maximal outcomes), the term in the context of Decision Making, Simon [25] suggests satisficing, which means that a decision-maker is looking for satisfactory outcomes rather than maximal outcomes in a decision situation. Ahituv and Wand [4] integrate this approach in the Information Structure Model, defining a satisfaction level. Outcomes above the satisfaction level obtain a value of 0 , while all outcomes under this level obtain a value of 1 in the outcome matrix. This model presents a decision situation as a dual problem to the outcome-maximizing problem, in which the possibility of obtaining unsatisfactory outcomes is minimized. Ahituv and Wand are able to solve the optimizing problem under the assumption of minimal risk.

The problematic issues preventing an accurate calculation of the value of information - specifically because of intangible benefits and the digital characteristics of information - were presented by Ahituv [1] [2]. This, in turn, led researchers to use information systems with tangible (quantitative) benefits [3] [14] [21].

Some normative models describe a multi-objective (preferences) function ("Multi Objective Utility Function" [11] [12]). By using these models, calculation functions and order rankings are defined. For example, Ronen and Spector [22] demonstrated a methodology for using the information structure model in order to adjust the sample group size and level of acceptance in quality control programs. This methodology weighs quantitative criteria in a linear manner: expected outcome and standard deviation. According to this research, the assumption is that a decision process is based on multi-decision criteria. Unfortunately, a decision-maker does not know how to integrate these criteria into a single utility function. Hence, the solution is not achieved through optimization, and a different approach is required.

Several studies examine the issue of MCDM (Multi Criteria Decision Making). Kabir et al. [10] identified and classified 300 researches in the field of infrastructure management, which are related to the field of MCDM. Monterio Gomes et al. [18] present a model for analysis of the behavioral aspects of MCDM. They also analyze the implications of using the TODIM method. Othman et al. [19] deals with the use of Geographical Information System (GIS) and Multi-criteria Decision Making. Durban and Stewart [9] present methods of MCDA (Multi Criteria Decision Analysis), where attribute evaluations are uncertain. Based on the Prospect Theory, Meng et al. [16] develop a method of the multi-attribute decision making using Atanassov's interval-valued intuitionistic fuzzy information. Rezaei [20] uses the Best Worst Method (BWM) and calculate a weight of each criterion in order to rank criteria from the most important criterion to the least important one, which are set up beforehand.

Thokala et al. [27] deal with the decision making processes and techniques in the healthcare sector which is characterized by a lot of intangible aspects. This research that accounts for uncertain attributes in the decision making during the design stage of DSS, may be implemented in the healthcare area as well.

The productivity paradox of ICT has been recognized for 
the last 30 years. Brynjolfsson [5] [6] and Brynjolfsson and Hitt [7] analyzed the attributes and the reasons for such phenomenon. Evidence showed that investment in ICT sometimes leads organizations in wrong directions. Moreover, ICT investment sometimes harms profits and income. Over the years, it has been understood that productivity measures are immature and further investigation to better understand ICT productivity measures is required [8]. Mithas et al. [17] identified a connection between IT productivity and revenue growth rather than between IT productivity and cost reduction. Tambe and Hitt [26] found that IT investment contributes to the success of large firms, since they invest with a long-range view. Kleis et al. [13] found a relationship between investment in IT and innovative productivity improvement (defined as intangible utility). The current research analyzes the contradiction between investment in IT and multi-utility function in a normative fashion.

\section{Modeling the Multi Criteria Decision Making Decision Environment}

Savage's [24] rationality assumptions are the basis for calculating the value of information by using the information structure model [15]. The $3^{\text {rd }}$ rationality assumption is based on a decision-makers' infinite calculation ability. Suppose (for the sake of simplicity) that this is true. Can the decision-maker integrate different aspects of utility with a comprehensive integrated utility function? When the answer to the above question is no, we should try to extend the information structure model to include a multi-criteria environment as is shown in Definition 1.

\subsection{Definition 1: Multi-criteria Rank Order}

Let $Q_{1}$ and $Q_{2}$ be two information structures operating on the same set of states of nature $S=\left\{S_{1}, \ldots, S_{n}\right\}$, and producing the same set of signals $Y=\left\{Y_{1}, \ldots, Y_{m}.\right\}$. Let $U$ be a utility matrix from the order rxn. Every utility has a k-dimensional vector.

$$
\forall i, i=1, . ., r, \forall j, j=1, . ., n, \quad U_{i, j}=(u 1, . ., u \mathrm{k}) \text {, and the } \mathrm{k} \text { aspects }
$$

(dimensions) are not calculable into a single utility function:

$$
(v 1, . ., v k) \geq(w 1, . ., w k) \Leftrightarrow \forall i, i=1, . ., k, v i \geq w i
$$

The implication is that when we compare two utility vectors, $V$ and $W, V$ is preferred over $W$ if and only if it is preferable for each and every dimension. This is a partial transitive ranking order, but a utility optimization does not always exist, like in the one-dimensional scenario. Hence, the multi-utility scenario is not a scenario in which we can always calculate an optimal solution.

Although Blackwell's theorem exists in the multi-utility environment, the solution to the multi-utility problem is not achieved by calculating an optimization problem; thus, another approach is required.
When the general information ratio [15] is examined in a multi-dimensional environment, in which the decision-maker is unable to integrate his or her benefits, then the basic definition of the Blackwell Theorem [15] is inadequate. This theorem compares the optimal expected value; however, this is not appropriate because if the decision-maker cannot weigh the expected benefits of the utility function, then it is not always possible to calculate the optimal solution. Determining the threshold criterion begins to affect the situation of a single decision by choosing a strategy that requires a number of considerations, some of which may be non-quantifiable regarding benefits and weighing. Let us assume the present situation of a decision with the following characteristics:

1. The utility of making a decision is multi-dimensional. The decision-maker has more than one consideration when making a decision.

2. There is no option to build an integrated utility function among the utility's components.

3. The decision-maker knows the rate of the probabilistic combination of benefits.

4. The decision-maker is of the EMV type.

Therefore, examining the general informativeness ratio using a different approach is required, as is shown below in Definition 2.

\subsection{Definition 2: Optimizer s.t. Rational Satisfaction}

A decision-maker is an optimizer subject to rational satisfaction.

Assume every item of $U$ contains a k-dimensional vector, in which each dimension represents intangible criteria. The optimization problem subject to rational satisfaction is calculated as follows:

$$
\begin{aligned}
& \underset{D}{\operatorname{Max}}\left(\operatorname{trace}\left(\Pi^{*} Q^{*} D^{*} U\right)\right)=(v 1, \ldots, v k) \\
& v=\min (v 1, \ldots, v k)
\end{aligned}
$$

In fact, it is a max-min approach. Decision-makers try to achieve a certain satisfaction level for each and every criterion.

$$
\underset{D}{\operatorname{Max}}\left(\operatorname{trace}\left(\Pi^{*} Q^{*} D^{*} U\right)\right) \geq v
$$

For example, suppose there are 3 dimensions. Using the max-min approach, the solution is as follows:

$$
\begin{aligned}
& \underset{D}{\operatorname{Max}}\left(\operatorname{trace}\left(\prod^{*} Q^{*} D^{*} U\right)\right)=(2,5,4) \\
& \text { Hence, } v=2
\end{aligned}
$$

In these cases, a conflict might arise between different considerations; hence, an optimal decision cannot be made. In these types of decision situations, a different approach is required in order to select the most preferable strategy. Suppose then, that the decision-maker is a typical Optimizer subject to Rational-Satisfaction interested in an alternative that will guarantee her the maximal of minimum level of the 
possible values for each criterion.

It can be argued that a decision-maker cannot integrate these criteria together; so, how can she make the decision? The basic assumption is that a decision-maker does not integrate these criteria. She assesses each and every one separately, and tries to achieve a satisfaction level for every criterion.

\section{The Dual Paradox, an Analysis of the Marginal Changes of Expected Utility, Subject to Equilibrium of Utility Dimensions}

\subsection{The Paradox - An analysis}

In order to identify a decision process of a decision-maker of the "optimizer subject to rational satisfaction" type, we analyze the "suspicious points" for determining this threshold level, and show that equilibrium always exists. It is shown below in Theorem1 that when one has to make a decision, in a situation where there are two criteria, an equilibrium point exists (in fact, the existence of this equilibrium point is proven in Theorem 1).

\section{Theorem 1}

Let $Q$ be an information structure operating on $S=$ $\{S 1, \ldots, S n\}$ a set of states of nature, and producing $Y=\{Y 1, \ldots, Y m$. $\}$, a set of signals. Let $U$ be a utility matrix from the order $2 \mathrm{xn}$. Let $\mathrm{r}$ be the number of possible decisions.

$$
\forall i, i=1, \ldots, r, \forall j, j=1, \ldots, n, \quad U_{i, j}=(v 1, v 2),
$$

and the aspects (dimensions) are not calculable:

$$
\left(v 1, v_{2}\right) \geq(w 1, w 2) \Leftrightarrow \forall i, i=1,2 \quad v_{i} \geq w \mathrm{i} .
$$

Let the decision-maker be of the "optimizer subject to rational satisfaction" type. Then, each signal has an optimal strategy combined of one or two pure strategies. Moreover, an equilibrium, which guarantees a unique (one and only one) set of outcomes, exists for the decision-maker so she will not deviate from this set. The proof appears in Appendix 1.

From the concept of Theorem 1, we can derive the mechanism of the following calculation:

Let $A$ land $A 2$ be two alternatives in a given decision situation. Let $U_{1}=\left(u 11, u 1_{2}\right)$, and $U_{2}=(u 21, u 22)$ be their two-dimensional vector expected utility function, accordingly. $U_{1}$ is not dominated by $U_{2}$ and vice-versa. Suppose, without losing generality, $u 1_{1}>u 2_{1}$ and $u 2_{2}>u 1_{2}$. Hence, a mixed strategy between $U_{1}$ and $U_{2}$ will provide equilibrium. Denote:

$$
\Delta U_{1}=u 1_{1}-u 1_{2}, \Delta U_{2}=u 2_{2}-u 2_{1}
$$

Let $W=p^{*} U_{1}+(1-p) * U_{2}$, a two-dimensional vector of expected outcomes of the equilibrium strategy, denoted as $(p$, 1-p). From this, it is derived that

$$
p=\frac{\Delta U_{2}}{\Delta U_{1}+\Delta U_{2}},
$$

where $\mathrm{p}$ is the probability to act $A 1$.

Let's now examine in Table 1, four possible expected utility change scenarios, without losing generality, supposing that the utility of U1, the action A1 was changed.

Scenarios 3 and 4 demonstrate a paradox, from the service suppliers' point of view. In a competitive market, if your client is a decision-maker of the "optimizer subject to rational satisfaction" type, the investments that have already gained a competitive advantage will lead to a decrease in the market share.

Table 1. Multi-Criteria Scenarios

\begin{tabular}{|c|c|c|}
\hline The Scenario & Mixed Strategy - the probability of $A 1$ & Meaning \\
\hline 1. $u 12$ increased by $\varepsilon$ & $p 1=\frac{\Delta U_{2}}{\left(\Delta U_{1-\varepsilon}\right)+\Delta U_{2}}>p$ & Normal \\
\hline 2. $u 12$ decreased by $\varepsilon$ & $p 2=\frac{\Delta U_{2}}{\left(\Delta U_{1}+\varepsilon\right)+\Delta U_{2}}<p$ & Normal \\
\hline 3. $u 11$ increased by $\varepsilon$ & $p 3=\frac{\Delta U_{2}}{\left(\Delta U_{1}+\varepsilon\right)+\Delta U_{2}}<p$ & Paradox \\
\hline $4 . \quad u 11$ decreased by $\varepsilon$ & $p 4=\frac{\Delta U_{2}}{\left(\Delta U_{1-\varepsilon}\right)+\Delta U_{2}}>p$ & Paradox \\
\hline
\end{tabular}




\subsection{The Paradox - An example}

Suppose that during a firm's preparation of an ICT master plan, the CIO (Chief Information Officer) can employ two consulting firms. The CIO uses two criteria in order to mitigate risks: orientation of innovation and the ability to cooperate with the business units. The CIO ranks the consulting firms using these criteria between 1 and 7 , as is shown below in Table 2 .

Table 2. Rank of firms - Initial state

\begin{tabular}{|c|c|c|}
\hline Criterion & Rank of Firm A & Rank of Firm B \\
\hline Innovation & 6 & 4 \\
\hline Cooperation & 4 & 6 \\
\hline
\end{tabular}

We demonstrate the paradox using two scenarios. Firm B improves its level of cooperation according to the first scenario and decreases its level of cooperation according to the second scenario.

First Scenario:

Since the CIO is a decision-maker of the "optimizer subject to rational satisfaction" type, the share of each firm is derived from Theorem 1and is calculated as follows:

$$
\operatorname{Max}-\operatorname{Min}[\mathrm{p} *(6,4)+(1-\mathrm{p}) *(4,6)] \Rightarrow \mathrm{p}=0.5
$$

Hence, the CIO divides the consulting services equally between the two consulting firms (a share of 50\% for each firm). Hence, the expected utility from her/his point of view for both criteria (Innovation and Cooperation) is 5 .

Three years later, the CIO needs to employ consulting services for another master plan. She wants to use Firm A and Firm B's consulting services. It is known that Firm A has retained the same level of innovation and cooperation, while Firm B has improved its level of cooperation. The firms' ranks are shown below in Table 3 .

Table 3. Rank of firms - First Scenario

\begin{tabular}{|c|c|c|}
\hline Criterion & Rank of Firm A & Rank of Firm B \\
\hline Innovation & 6 & 4 \\
\hline Cooperation & 4 & 7 \\
\hline
\end{tabular}

Since the CIO is a decision-maker of the "optimizer subject to rational satisfaction" type, the share of each firm is derived from Theorem 1 and is calculated as follows:

$$
\operatorname{Max}-\operatorname{Min}\left[\mathrm{p} *(6,4)+(1-\mathrm{p})^{*}(4,7)\right] \Rightarrow \mathrm{p}=0.6
$$

That is, the CIO orders $60 \%$ of the work from Firm A, and $40 \%$ from Firm B. Now, the expected utility for both criteria (Innovation and Cooperation) is 5.2 that is higher than 5 in the initial state. Figures 1 illustrate these results. Figure 1a (the upper figure) presents the expected utility of the decision maker for each criterion in the initial state and Figure $1 \mathrm{~b}$ (the lower figure) presents the expected utility of the decision maker three years later as functions of Firm A's share, $p$. The two lines intersect at the equilibrium point, which constitutes the optimal share of Firm A from the decision maker's point of view. Thus, the share of Firm B, $1-p$, decreases from $50 \%$ to $40 \%$, as shown in Figures 1, though its cooperation level has increased.
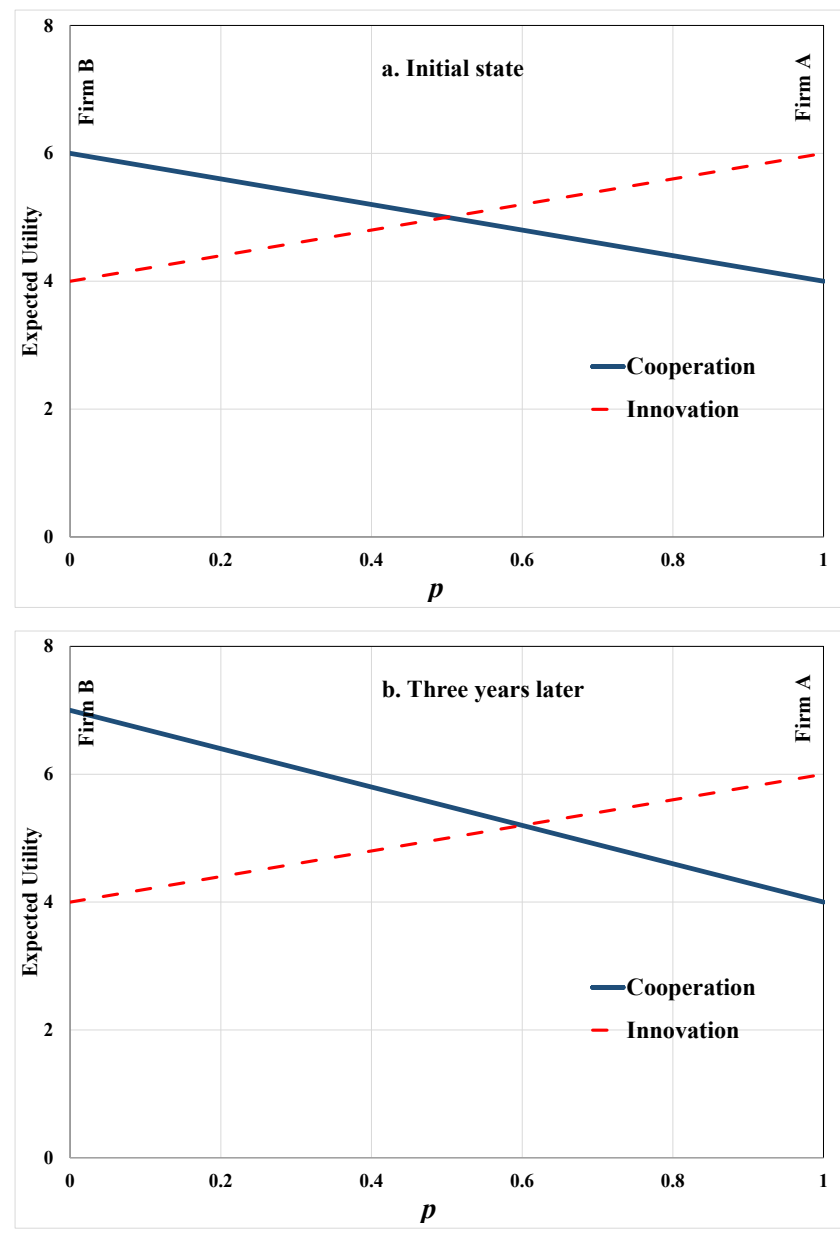

Figure 1. Illustration of the first scenario

The first scenario demonstrates the productivity paradox from the supplier's point of view. In a competitive market, if a client is a decision-maker of the "optimizer subject to rational satisfaction" type, the investments that have already gained a competitive advantage may lead to a decrease in the market share.

Second Scenario:

In this scenario the CIO faces the same initial state (see Table 2). However, three years later, when the CIO requires consulting services for another master plan (and she wants to use Firm A and Firm B's consulting services), we assume that Firm A has maintained the same level of innovation and cooperation, while Firm B's cooperation level has been decreased. The firms' rankings are presented below in Table 4.

Table 4. Rank of firms - Second Scenario

\begin{tabular}{|c|c|c|}
\hline Criterion & Rank of Firm A & Rank of Firm B \\
\hline Innovation & 6 & 4 \\
\hline Cooperation & 4 & 5 \\
\hline
\end{tabular}

Since the CIO is a decision-maker of the "optimizer subject to rational satisfaction" type, the share of each firm is derived from Theorem 1 and is calculated as follows: 


$$
\operatorname{Max}-\operatorname{Min}\left[p^{*}(6,4)+(1-p) *(4,5)\right] \Rightarrow p=1 / 3
$$

That is, the CIO orders $1 / 3$ of the work from Firm A, and $2 / 3$ from Firm B. Now, the expected utility for both criteria (Innovation and Cooperation) is 4.67 that is lower than 5 in the initial state. Figures 2 illustrate these results. Figure $2 \mathrm{a}$ (the upper figure) presents the expected utility of the decision maker for each criterion in the initial state and Figure $2 b$ (the lower figure) presents the expected utility of the decision maker three years later as functions of Firm A's share, $p$. The two lines intersect at the equilibrium point, which constitutes the optimal share of Firm A from the decision maker's point of view. Thus, the share of Firm B, $1-p$, increases from $50 \%$ to $66.7 \%$, as shown in Figures 2, though its cooperation level has decreased.
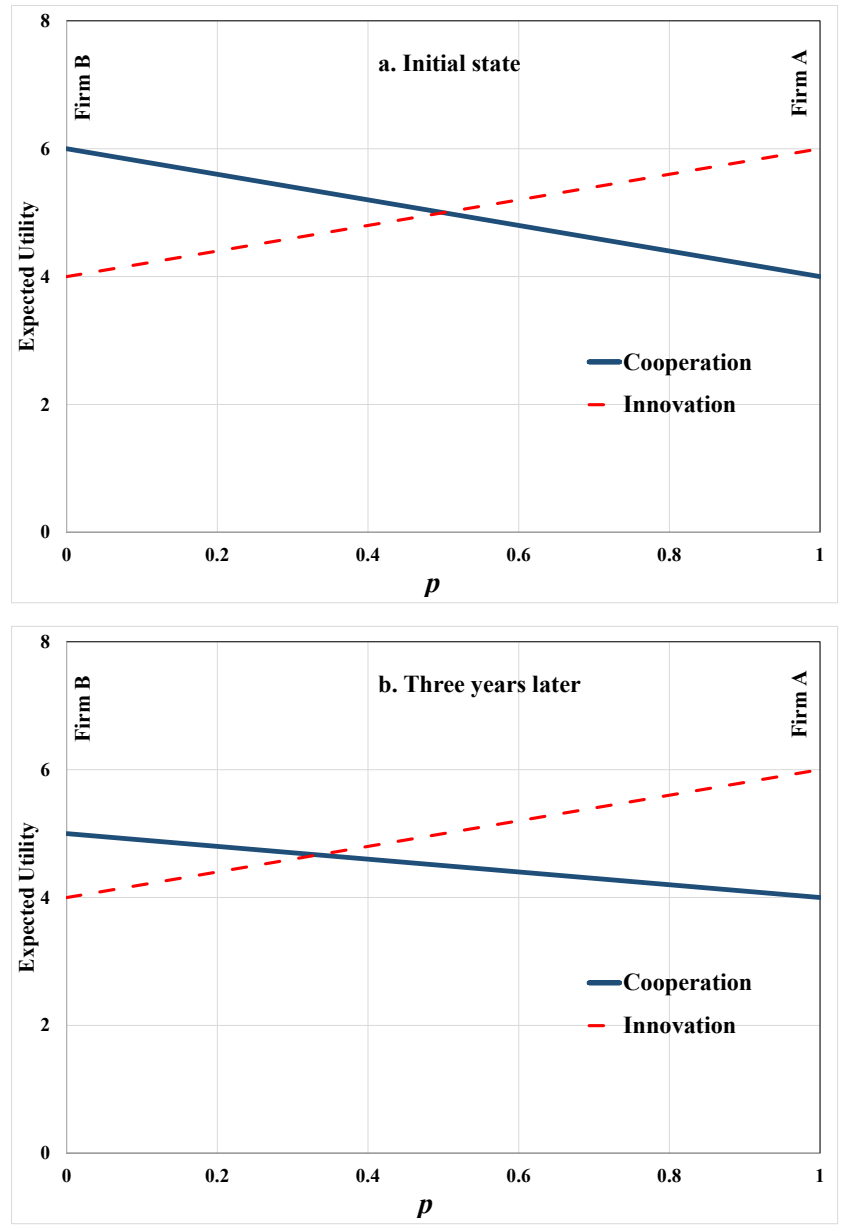

Figure 2. Illustration of the second scenario
The second scenario demonstrates the productivity paradox from the supplier's point of view: a decrease in the level of service that reduces a firm's competitive advantage, may lead to a paradoxical market share increase, if its client is a decision-maker of the "optimizer subject to rational satisfaction" type.

\section{Summary and Conclusions}

The current paper established an integrated, normative framework for an analysis of the multi-criteria decision-making process, by taking into consideration the following aspects: Intangible criteria, Bounded Rationality, a Multi-Dimensional Utility function, and the General Informativeness ratio. The proposed method may be useful for better understanding of several decision making situations, when intangible decision criteria are taken into consideration, for example, a decision on a content of new type of food product by weighting tastes, flavors, and healthfulness. Another example is in the healthcare area, when a patient should decide on a treatment by weighting safety, level of recovery, trends, and reputation.

The paradox which is demonstrated (in section 4.2), can explain several scenarios in the development of ICT products. Sometimes, when a client mixes/integrates/combines information technology products, an improvement can lead to failure. This can explain why superior technologies sometimes fail. Moreover, it seems that in certain scenarios it is better to supply less productivity in a strong area when a company has a competitive advantage. It is assumed that these "bounded rationality" considerations can harm productivity.

Moreover, this research could lead to a better understanding of decision-making processes over time. This could then lead to improved decision outcomes, for example, on the performance of DSS which deal with MCDM scenarios.

Further research could be in order to better analyze and understand the value of a second opinion in terms of the Multi-Criteria issues. Moreover, an analysis of the equilibrium in a "k criteria" environment is required. Furthermore, empiric research may provide better understanding of the implementation of this approach. 


\section{Appendix}

\section{Theorem 1}

Let $Q$ be an information structure operating on $S=\{S 1, \ldots, S n\}$ a set of states of nature, and producing $Y=\{Y 1, \ldots, Y m\}$, a set of signals. Let $U$ be a utility matrix from the order $2 \mathrm{xn}$. Let $\mathrm{r}$ be the number of possible decisions.

$\forall i, i=1, . ., r, \forall j, j=1, . ., n, U_{i, j}=(v 1, v 2)$, and the aspects (dimensions) are not calculable:

$(v 1, v 2) \geq(w 1, w 2) \Leftrightarrow \forall i, i=1,2 \quad v_{i} \geq w \mathrm{i}$

Let the decision-maker be of the "optimizer subject to rational satisfaction" type.

Then, each and every signal has an optimal strategy combined of one or two pure strategies. Moreover, an equilibrium, which guarantees a unique (one and only one) set of outcomes, exists for the decision-maker so she will not deviate from this set.

\section{Proof}

(1) Let's solve the optimization problem of the information structure model separately for each utility dimension. Since there are 2 non-integrative utility dimensions, we will calculate the optimal decision rule separately for each dimension. Since there are $\mathrm{r}$ decisions, every signal has 2 dimension vectors.

$$
\text { Denoted Vi }, \forall i, i=1, . ., r, \quad V_{i}=(v 1, v 2)_{i}
$$

(2) First, inferior (dominated) strategies, have been omitted. $V_{i}=(v 1, v 2)_{i}$ is a strategy that is being dominated if

$$
\exists V_{j}=(v 1, v 2)_{j},(v 1, v 2)_{j} \geq(v 1, v 2)_{i} \Leftrightarrow\left((v 1)_{j} \geq(v 1)_{i}\right) \text { and }\left((v 2)_{j} \geq(v 2)_{i}\right)
$$

(While there are two (or more) equivalent strategies, one of them remains. It is obvious that at least one strategy is not being dominated subject to this algorithm).

(3) In this stage, $\mathrm{r} 1$ strategies exist, $\mathrm{r} 1 \leq \mathrm{r}$. For the sake of simplicity, let's rearrange them

$$
\forall i, i=1, . ., r 1, \quad V_{i}=(v 1, v 2)_{i}
$$

(4) Let's check if between the two dimensions there is a dominant dimension (meaning, any alternative's expected utility from the superior dimension is at least equal to the expected utility of the inferior dimension).

$$
\forall i, i=1, . ., r 1, \quad\left((v 1)_{i} \geq(v 2)_{i}\right) \text { or } \forall i, i=1, . ., r 1,\left((v 1)_{i} \leq(v 2)_{i}\right)
$$

(5) In fact, if one of the dimensions is dominant, there is a pure strategy solution based on the dominated (inferior) dimension (an alternative that guarantees maximal utility of that dimension).

(6) Let's examine the situation in which there is no dominated dimension. For the sake of convenience, the strategies have been denoted $V_{1}, . . V_{r 1}$. Let's check whether the following exists for every two alternatives:

$$
\begin{aligned}
& \left(\forall i=1, . ., r 1, \exists j=1, . ., r 1, i \neq j, \quad\left((v 1)_{i} \leq(v 2)_{i}\right) \quad \text { and }\left((v 1)_{j} \leq(v 2)_{j}\right) \quad \text { and }\left((v 1)_{i} \leq(v 1)_{j}\right)\right) \\
& \text { or } \\
& \left(\forall i=1, . ., r 1, \exists j=1, . ., r 1, i \neq j, \quad\left((v 1)_{i} \geq(v 2)_{i}\right) \quad \text { and }\left((v 1)_{j} \geq(v 2)_{j}\right) \text { and }\left((v 2)_{i} \leq(v 2)_{j}\right)\right)
\end{aligned}
$$

(7) That between these two alternatives there is a dominated dimension. In this case, the alternative $\mathrm{j}$ is preferred over $\mathrm{i}$. Later, it is proven that except in the scenario $\left((v 1)_{j}=(v 2)_{j}\right)$, equilibrium does not exist between alternatives $\mathrm{i}$ and $\mathrm{j}$.

(8) When the scenario is:

$$
\left(\left((v 1)_{i}<(v 2)_{i}\right) \text { and }\left((v 1)_{j}>(v 2)_{j}\right)\right) \text { or }\left(\left((v 1)_{i}>(v 2)_{i}\right) \text { and }\left((v 1)_{j}<(v 2)_{j}\right)\right)
$$

(9) We will solve the following equation:

$$
\begin{gathered}
p^{*}(v 1)_{i}+(1-p)^{*}(v 1)_{j}=p^{*}(v 2)_{i}+(1-p)^{*}(v 2)_{\mathrm{j}} \Leftrightarrow \\
p^{*}\left((v 1)_{i}-(v 1)_{j}+(v 1)_{j}-(v 2)_{i}\right)=(v 2)_{j}-(v 1)_{j} \Rightarrow \forall i=1, . ., r 1, \forall j=1, . ., r 1, i \neq j, \quad p=\frac{(v 2)_{j}-(v 1)_{j}}{\left((v 1)_{i}-(v 1)_{j}+(v 2)_{j}-(v 2)_{i}\right)}
\end{gathered}
$$

(10) Without losing generality, suppose $\left(v_{2}\right)_{j}-\left(v_{1}\right)_{j}>0$, then: 


$$
p=\frac{(v 2)_{j}-(v 1)_{j}}{\left((v 1)_{i}-(v 1)_{j}+(v 2)_{j}-(v 2)_{i}\right)} \Rightarrow 0<p<1
$$

(11) Moreover, the following equation exists:

$$
\min (v 1, v 2)_{i} \leq p^{*}(v 1)_{i}+(1-p) *(v 1)_{j}=p^{*}(v 2)_{i}+(1-p) *(v 2)_{j} \geq \min (v 1, v 2)_{j}
$$

(12) Therefore, there are $\frac{r 1^{*}(r 1-2)}{2}$ possible solutions (number of foreign pairs of alternatives).

It will be shown that in a situation where a dominant pure strategy does not exist, and a dominant dimension also does not exist, the equilibrium solution exists, and is a mixture of pure strategies (in fact, 2 strategies).

(13) Let's examine each pair of strategies that fulfills the criteria:

$$
\begin{aligned}
& \left(\forall i=1, . ., r 1, \exists j=1, . ., r 1, i \neq j, \quad\left((v 1)_{i} \leq(v 2)_{i}\right) \text { and }\left((v 1)_{j} \leq(v 2)_{j}\right) \text { and }\left((v 1)_{i} \leq(v 1)_{j}\right)\right) \\
& \text { or } \\
& \left(\forall i=1, . ., r 1, \exists j=1, . ., r 1, i \neq j, \quad\left((v 1)_{i} \geq(v 2)_{i}\right) \text { and }\left((v 1)_{j} \geq(v 2)_{j}\right) \text { and }\left((v 2)_{i} \leq(v 2)_{j}\right)\right)
\end{aligned}
$$

(There is a dominated dimension between these two strategies).

(14) $\operatorname{If}\left((v 1)_{j}=\left(v_{2}\right)_{j}\right)$, the $\mathrm{j}$ strategy could be an equilibrium strategy.

It will be proven that if $\left(\left(v_{1}\right)_{j} \neq\left(v_{2}\right)_{j}\right)$, then strategy $" \mathrm{j}$ " is not an equilibrium strategy.

(15) Since there is no dominated dimension, there is a "v" strategy,

$$
\begin{aligned}
& \exists v=1, . ., r 1, v \neq i, v \neq j, \\
& \left(\left((v 1)_{v}<(v 2)_{v}\right) \text { and }\left((v 1)_{j}>(v 2)_{j}\right)\right) \text { or }\left(\left((v 1)_{v}>(v 2)_{v}\right) \text { and }\left((v 1)_{j}<(v 2)_{j}\right)\right)
\end{aligned}
$$

(16) From (11) it is derived that a mixed strategy exists (between strategy " $v$ " and strategy "j") which a decision-maker will prefer over strategy "j".

(17) Hence, a mixed strategy is feasible and there can only be one pure strategy with $s \leq \frac{r 1^{*}(r 1-2)}{2}$ elements.

(18) $W_{i}$ is denoted as the two dimensions' expected outcomes, following this strategy

$\forall i=1, . . s \quad W_{i}=\left(w 1, w_{2}\right)_{i}, w 1=w 2$. Following this, we denote $W_{a}$ as a dominant strategy that a decision-maker would prefer most.

(19) Suppose (for the sake of contradiction) that there is a mixed strategy comprised of more than 2 pure strategies, which are not dominated. $Z=(z 1, z 2)=\sum_{i} p_{i} * V_{i}, \quad z 1=z 2 \geq(w 1)_{a}=(w 2)_{a}$

(20) Let's divide $Z$ into three sub-sets $\hat{V}_{1}=\left\{V_{i} \mid v_{1}>v_{2}\right\}, \hat{V}_{2}=\left\{V_{i} \mid v_{1}<v_{2}\right\}, \hat{V}_{3}=\left\{V_{i} \mid v_{1}=v_{2}\right\}$

(21) If $V_{i} \in \hat{V}_{3}$ then $V_{i}=W_{a}$; otherwise, there is another preferable strategy (contradiction).

(22) Otherwise, $\left(\hat{V}_{3}=\varphi\right)$. Hence, strategy $Z$ can be represented as a combination of mixed strategies, each of which is a convex combination of pure strategies from $\hat{V}_{1}$ and $\hat{V}_{2}$, respectively.

(23) Hence: $Z=\sum_{i} q_{i} * W_{i}$, and $Z=\sum_{i} q_{i} * W_{a}$.

(24) Thus, for any given signal only a pure strategy or a mixed strategy of two pure strategies can be chosen. This is the case for each and every signal.

Q.E.D. 


\section{REFERENCES}

[1] N. Ahituv, A Systematic Approach toward Assessing the Value of an Information System, MIS Quarterly. 4(4)) (1980) 61-75.

[2] N. Ahituv, Assessing the Value of Information: Problems and Approaches, Proceedings of ICIS-89. (1989) 315-325.

[3] N. Ahituv, and G. Greenstein, Systems Inaccessibility and the Productivity Paradox, European Journal of Operational Research. 161 (2005) 505-524.

[4] N. Ahituv, and Y. Wand, Comparative Evaluation of Information under Two Business Objectives, Decision Sciences. 15(1) (1984) 31-51.

[5] E. Brynjolfsson, The Productivity Paradox of Information Technology, Communications of the ACM. 36(12) (1993) 6777.

[6] E. Brynjolfsson, Paradox Lost, CIO. 7(14) (1994) 26-28.

[7] E. Brynjolfsson, and L. M. Hitt, Beyond the Productivity Paradox, Communications of the ACM. 41(8) (1998) 49-55.

[8] Y. C. Chou, H. H. C. Chuang, and B. B. M. Shao, The impacts of information technology on total factor productivity: A look at externalities and innovations. International, Journal of Production Economic. 158 (2014) 290-299.

[9] I. N. Durban, and T. J. Stewart, Modeling uncertainty in multi-criteria decision analysis, European Journal of Operations Research. 223 (2012) (1).1-14.

[10] G. Kabir, R. Sadiq, and S. Tefamariam, A review of multi-criteria decision-making methods for infrastructure management, Structure and Infrastructure Engineering: Maintenance, Management, Life-Cycle Design and Performance. 10 (9) (2014) 1176-1210.

[11] R. L. Keeney, Value-focused thinking: Identifying decision opportunities and creating alternatives, EJOR. 92(3) (1992) 537-549.

[12] R. L. Keeney, and H. Raiffa, Decisions with Multiple Objectives: Preferences and Value Trade-Offs, (Cambridge University Press, UK, 1993).

[13] L. Kleis, P. Chwelos, R. V. Ramirez and I. Cockburn, Information Technology and Intangible Output: The Impact of IT Investment on Innovation Productivity, Information Systems Research. 23(1) (2011) 42-59.

[14] N. Margaliot, Selecting a Quality Control Attribute Sample: An Information-Economics Method, Annals of Operations Research. 91 (1999) 83-104.

[15] C. B. McGuire and R. Radner, R. (editors) (1986). Decision and Organization (ch. 5), Minneapolis, Minnesota: University of Minnesota Press, 2nd edition.

[16] F. Meng, C. Tan, and X. Chen, An approach to Atanassov's interval-valued intuitionistic fuzzy multi-attribute decision making based on prospect theory, International Journal of Computational Intelligence Systems. 8(3) (2015) 591-605.

[17] S. Mithas, A. R. Tafni, I. Bardhan, and J.M. Goh, Information Technology and Firm Profitability: Mechanisms and Empirical Evidence, MIS Quarterly. 36(1) (2012) 205-224.

[18] L. F. A. Monterio Gomes, M. A. Soares Machado, and L. A. Duncan Rangel, Behavioral multi-criteria decision analysis: the TODIM method with criteria interactions, Annals of Operations Research. 211(1) (2013) 531-548.

[19] M.Othman, W.H. Naim, and S. Noraisi, GIS Based Multi-Criteria Decision Making for Landslide Hazard Zonation, Procedia - Social and Behavioral Sciences. 35 (2012) 595-602.

[20] J. Rezaei, Best-worst multi-criteria decision-making method, Omega. 53 (2015) 49-57

[21] B. Ronen, An Information-Economics Approach to Quality Control Attribute Sampling, European Journal of Operational Research. 73 (1994) 430-442

[22] B. Ronen, and Y. Spector, Theory and Methodology Evaluating Sampling Strategy under Two Criteria, European Journal of Operational Research. 80 (1995) 59-67.

[23] Rubinstein, Modeling Bounded Rationality (Cambridge, Massachusetts: M.I.T Press., 1998)

[24] L. J. Savage. The Foundation of Statistics, John Wiley \& Sons Inc., New-York, 1954.

[25] H. A. Simon, The New Science of Management Decisions, Harper and Row, New-York, 1960.

[26] P. Tambe, and L. M. Hitt, The Productivity of Information Technology Investments: New Evidence from IT Labor Data. Information Systems Research, 23(3) (2012) 599-617.

[27] P. Thokala, N. Devlin, K. Marsh, R. Baltussen, M. Boysen, Z. Kalo, T. Longrenn, F.Mussen, S. Peacock, J. Watkins, M. Ijzerman, Multiple Criteria Decision Analysis for Health Care Decision Making-An Introduction: Report 1 of the ISPOR MCDA Emerging Good Practices Task Force, Value in Health. 19 (1) (2016) 1 - 13 